\title{
Design of Flight Control System for a Novel Tilt-Rotor UAV
}

\author{
Zaibin Chen $\mathbb{D}^{1,2}$ and Hongguang Jia $\mathbb{D}^{1,2,3}$ \\ ${ }^{1}$ Changchun Institute of Optics, Fine Mechanics and Physics, Chinese Academy of Sciences, No. 3888, Dongnanhu Rd., \\ Changchun 130033, Jilin, China \\ ${ }^{2}$ University of Chinese Academy of Sciences, No. 19, Yuquan Rd., Beijing 10049, China \\ ${ }^{3}$ Chang Guang Satellite Technology Co., Ltd., No. 1299, Mingxi Rd., Changchun 130102, Jilin, China
}

Correspondence should be addressed to Zaibin Chen; chenzaibin14@mails.ucas.edu.cn

Received 29 November 2019; Revised 2 February 2020; Accepted 15 February 2020; Published 13 March 2020

Academic Editor: Cornelio Posadas-Castillo

Copyright (c) 2020 Zaibin Chen and Hongguang Jia. This is an open access article distributed under the Creative Commons Attribution License, which permits unrestricted use, distribution, and reproduction in any medium, provided the original work is properly cited.

\begin{abstract}
This paper presents the control system design process of a novel tilt-rotor unmanned aerial vehicle (TRUAV). First, a new configuration scheme with the tilting rotors is designed. Then, the detailed nonlinear mathematical model is established, and the parameters are acquired from designed experiments and numerical analyses. For control design purposes, the dynamics equation is linearized around the hovering equilibrium point, and a control allocation method based on trim calculation is developed. To deal with the actuator saturation and uncertain disturbance problems for the novel TRUAV, an improved flight control law based on the combination of the robust servo linear quadratic regulator (RSLQR) optimal control and the extended state observer (ESO) is proposed. The designed flight control law has a simple structure with a high reliability in engineering. Simulations and hovering flight tests are carried out to verify the effectiveness of the mathematical model and the proposed control strategy.
\end{abstract}

\section{Introduction}

Fixed-wing unmanned aerial vehicles (FWUAVs) and rotorcraft unmanned aerial vehicles (RUAVs), as representations of aircraft with conventional structures, have played important roles in military and civil fields for a long time. However, their applications are always limited by flexibility, payload, and endurance, which are concerned with their own typical structures and dynamical systems. To break the abovementioned limitations, the tilt-rotor UAVs (TRUAVs) have become a focus of UAV research [1]. Compared with RUAVs, the TRUAVs can be used in the scenarios that require higher speeds, longer flight ranges, and larger payload capacities. Compared with FWUAVs, the TRUAVs can perform take-off and landing (VTOL) flights without requiring a runway. Therefore, the TRUAVs have a wider range of applications than the other types of aircraft. A large proportion of the early studies on such aircraft focus on dual tilt-rotors such as Bell Eagle Eye [2], Smart UAV of KARI [3], and BIROTAN [4]. The Dual-TRUAVs have two tiltable rotors installed on the wingtips. Similar structure was also developed by China's Nanjing University of Aeronautics and Astronautics [5]. The Dual-TRUAVs generally require cyclic control of the propellers, which increases the mechanical complexity for stabilization and maneuverability. With the development of UAV design and control technology, some novel TRUAV structures have been explored in the past decade. In [6], an overactuated quadcopter was designed, which allows independent tilting of the rotors around their arm axes. This new structure improves the mobility and trajectory tracking capabilities by achieving full authority on torque and force vectoring. Similar structure was also displayed in reference [7]. In [8,9], a quad tilt-wing UAV was designed and constructed. It is a novel tilt-rotor structure in which rotors are assembled at the center of wings, and rotors and wings can tilt together to reduce the impact caused by the downwash. Papachristos et al. [10] adopted a tri-tilt rotor configuration that separates rotors and wings and consists of the prepositive double tilt rotors and a postpositive single tilt rotor. To reduce aerodynamic interference, rotors are mounted on both sides of the fuselage in front of and behind the wings. In [11], a new fixed-wing VTOL UAV structure 
was proposed, in which two tilting rotors are installed in front of the wings and another two rotors are positioned for coaxial main lift. Flores et al. $[12,13]$ developed a QuadTRUAV that has two pairs of tiltable rotors mounted at the front and back of the fuselage to reduce aerodynamic interference.

After evaluating the advantages and disadvantages of the different types of UAVs, a new structure scheme of the TRUAV is presented in this paper. The prototype is shown in Figure 1. Similar to the Quad-TRUAV, it has four rotors that are mounted at the front and back of the fuselage to implement all flight modes. The difference is that the new configuration only uses two tiltable rotors to meet thrust requirements, which means fewer mechanisms, lower cost, more energy savings, and longer flight durations. The propulsion systems 3 and 4 can tilt from the vertical position $\left(\varepsilon=0^{\circ}\right)$ to horizontal position $\left(\varepsilon=90^{\circ}\right)$ or inversely through a four-linkage mechanism. When the aircraft enters the fixedwing mode, the front rotors 1 and 2 stop operating; meanwhile, rotors 3 and 4 power the aircraft. Unlike the abovementioned UAVs, the new configuration adopts smaller motors and rotors for propulsion systems 3 and 4 to meet the low thrust-weight ratio requirement during the cruise flight, which reduces the aircraft weight and improves the cruising power efficiency. However, to ensure a sufficient pull during the VTOL phase, the propulsion systems 3 and 4 should have a longer moment arm, which brings greater challenges to the control system design. After repeated engineering estimation and total weight accounting, the design parameters of the aircraft are finalized. The main parameters are listed in Table 1 , in which $\bar{b}$ represents the span, $\bar{s}$ is the wing area, and $\bar{c}$ is the mean aerodynamic chord.

The ability to realize multimode flight brings about many benefits. However, as a specific flight vehicle, the TRUAV has more complex features than traditional aircraft, which makes it a highly nonlinear and coupling system with model uncertainties and unknown external disturbances and increases the complexity of controller design.

Considering these challenges, many intelligent nonlinear control methods have been investigated, such as the sliding mode control [14], the adaptive neural network control [15], the dynamic inversion method [16], and the neural network augmented model inversion control [17]. However, most related studies on the TRUAV focus on the simulation phase. It is very expensive and difficult to achieve these nonlinear control techniques in real flight tests because of the complexity of the design procedure, the considerable online computational burden and their strong dependence on the accuracy of nonlinear models. In engineering practice, the PID control is still well recognized. Yuksek et al. [18] successively designed a PID controller for the roll, pitch, and yaw channels, and the validity was verified by a flight experiment. Similar studies can also be found in the recent references $[19,20]$. However, the PID method has an unsatisfactory control performance and cannot deal with the actuator saturation and uncertain disturbance problems that often occur in the actual flight tests. Thus, how to design a better linear controller to solve these practical problems and improve the control performance motivates this work.

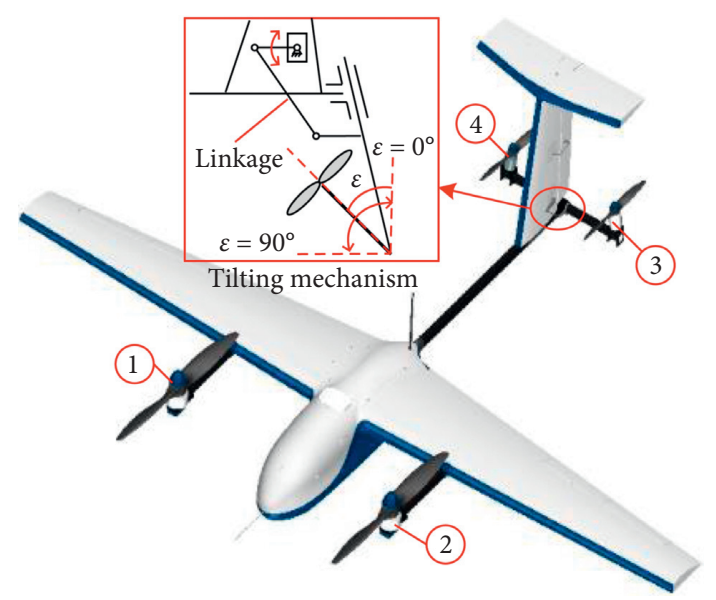

FIgURE 1: The structural platform of TRUAV.

TABLE 1: UAV parameters.

\begin{tabular}{lccc}
\hline Parameter & Value (unit) & Parameter & Value (unit) \\
\hline$m$ & $15.5 \mathrm{~kg}$ & $J_{r 1}\left(J_{r 2}\right)$ & $0.009 \mathrm{~kg} \times \mathrm{m}^{2}$ \\
$\bar{b}$ & $3.06 \mathrm{~m}$ & $J_{r 3}\left(J_{r 4}\right)$ & $0.001 \mathrm{~kg} \times \mathrm{m}^{2}$ \\
$\bar{s}$ & $1.04 \mathrm{~m}^{2}$ & $d_{1 x}\left(d_{2 x}\right)$ & $0.47 \mathrm{~m}$ \\
$\bar{c}$ & $0.36 \mathrm{~m}$ & $d_{1 y}\left(-d_{2 y}\right)$ & $-0.5 \mathrm{~m}$ \\
$I_{x x}$ & $2.14 \mathrm{~kg} \times \mathrm{m}^{2}$ & $d_{1 z}\left(d_{2 z}\right)$ & $-0.042 \mathrm{~m}$ \\
$I_{y y}$ & $5.45 \mathrm{~kg} \times \mathrm{m}^{2}$ & $d_{3 x}\left(d_{4 x}\right)$ & $-1.41 \mathrm{~m}$ \\
$I_{z z}$ & $7.18 \mathrm{~kg} \times \mathrm{m}^{2}$ & $d_{3 y}\left(-d_{4 y}\right)$ & $0.254 \mathrm{~m}$ \\
$I_{x z}$ & $0.56 \mathrm{~kg} \times \mathrm{m}^{2}$ & $d_{3 z}\left(d_{4 z}\right)$ & $-0.035 \mathrm{~m}$ \\
\hline
\end{tabular}

First, to protect the actuator from saturation and improve the control performance, a robust servo linear quadratic regulator (RSLQR) control method was developed in some other applications [21, 22]. In [21], this method was designed for the pitch angle control of a fixed-wing aircraft. Simulation results demonstrated that this method has a smaller control surface deflection than the conventional PID control in the initial response. In [22], the RSLQR control was applied to the pitch control of the missile autopilot. It exhibits a better tracking performance than the PID control by introducing a state error into the system. Thus, the RSLQR control method can solve the actuator saturation problem and improve the control performance. However, it cannot deal with the uncertain disturbance problems in the actual flight. To improve the antijamming ability of the control system, the RSLQR needs to be improved. In recent years, disturbance observers have been widely studied for some types of real-time systems. By using the observer properly, the disturbance rejection performance of the control system can be significantly improved. In particular, the ESO, known as the key module of active disturbance rejection control, is used to estimate system states and total disturbance with less dependence on model information $[23,24]$. The total disturbance mainly includes model uncertainty and external disturbance. It is treated as an additional state variable of the process and can be estimated online by the observer. In view of that an improved flight control law based on the combination of RSLQR with ESO is proposed to control the flight of the new TRUAV. Similar to the PID control, the designed controller is a linear control method with the features of low cost and easy realization. 
Furthermore, it solves the actuator saturation and uncertain disturbance problems and improves the control performance in the actual flight. Therefore, the proposed flight control law is prospective in the engineering application of the TRUAV.

The rest of the paper is organized as follows. In Section 2, the mathematical model is established and the related parameters are identified by designed experiments and numerical analyses. A control allocation method of the new TRUAV is developed, and a new flight control law based on the combination of RSLQR with ESO is proposed in Section 3. Then, simulations and flight tests are carried out in Sections 4 and 5. Finally, Section 6 concludes this paper and provides suggestions of future work.

\section{Mathematical Modeling}

The definitions of the main wing-body reference frames and corresponding conversion matrices can be found in most literature on aircraft dynamics [25]. Let $O_{b} x_{b} y_{b} z_{b}$ be the body-fixed frame (BFF) attached to the center of gravity (CoG) of the aircraft. The inertial earth-fixed frame (EFF) is expressed as $O_{g} x_{g} y_{g} z_{g}$ with its origin at the setting starting point. In addition, different from previous works, four auxiliary frames (AFs) $O_{i} x_{t i} y_{t i} z_{t i}(i=1,2,3,4)$ are built considering the tilting angle and the side inclination angle, where $z_{t i}$ points down along the propeller shaft, $x_{t i}$ is parallel to the longitudinal plane of the UAV, perpendicular to the $z_{t i}$ and pointing to the nose, and $y_{t i}$ is determined by the right-hand rule. The reference coordinate frames are shown in Figure 2. According to the principle of coordinate transfer, the transformation matrices among the coordination frames are given by

$$
\begin{aligned}
R_{g}^{b} & =\left[\begin{array}{ccc}
c \theta c \psi & c \theta s \psi & -s \theta \\
s \theta c \psi s \phi-s \psi c \phi & s \theta s \psi s \phi+c \psi c \phi & c \theta s \phi \\
s \theta c \psi c \phi+s \psi s \phi & s \theta s \psi c \phi-c \psi s \phi & c \theta c \phi
\end{array}\right], \\
R_{t 1}^{b} & =\left[\begin{array}{ccc}
1 & 0 & 0 \\
0 & c \eta & -s \eta \\
0 & s \eta & c \eta
\end{array}\right], \\
R_{t 2}^{b} & =\left[\begin{array}{ccc}
1 & 0 & 0 \\
0 & c \eta & s \eta \\
0 & -s \eta & c \eta
\end{array}\right], \\
R_{t 3}^{b} & =\left[\begin{array}{ccc}
c \varepsilon & s \varepsilon s \eta & -s \varepsilon c \eta \\
0 & c \eta & s \eta \\
s \varepsilon & -c \varepsilon s \eta & c \varepsilon c \eta
\end{array}\right], \\
R_{t 4}^{b} & =\left[\begin{array}{ccc}
c \varepsilon & -s \varepsilon s \eta & -s \varepsilon c \eta \\
0 & c \eta & -s \eta \\
s \mathcal{E} & c \varepsilon s \eta & c \varepsilon c \eta
\end{array}\right],
\end{aligned}
$$

where $\phi, \theta$, and $\psi$ represent the Euler angles, $c$ and $s$ are symbols for the cosine and sine functions, respectively, $\eta$ is the fixed lateral inclination angle introduced to the propulsion systems to improve the system controllability [26], and $\varepsilon$ is the tilting angle of the back rotors.

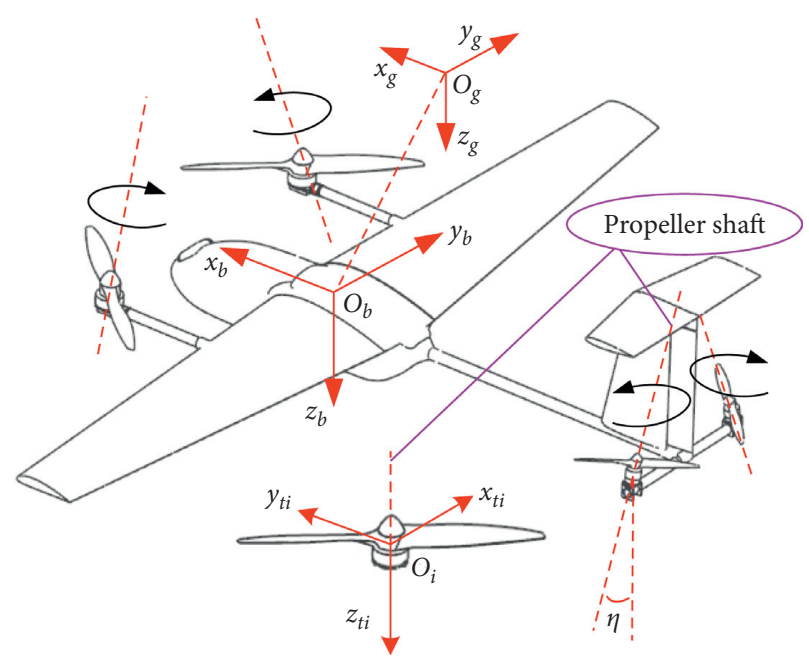

FIgURE 2: TRUAV structure and coordinate system.

The nonlinear dynamic model of the TRUAV can be derived in the form of a Newton-Euler formulation, which is expressed in the BFF as follows:

$$
\begin{aligned}
m \dot{V}^{b}+\omega^{b} \times V^{b} & =F^{b}, \\
I_{b} \dot{\omega}^{b}+\omega^{b} \times I_{b} \omega^{b} & =M^{b},
\end{aligned}
$$

where $m$ is the aircraft mass, $F^{b}=\left[\begin{array}{lll}F_{x}^{b} & F_{y}^{b} & F_{z}^{b}\end{array}\right]^{T}$ and $M^{b}=$ $\left[\begin{array}{lll}M_{x}^{b} & M_{y}^{b} & M_{z}^{b}\end{array}\right]^{T}$ represent the total force and moment vectors expressed in the BFF, respectively, $V^{b}=\left[\begin{array}{lll}u & v & w\end{array}\right]^{T}$ is the velocity vector in the BFF, $\omega^{b}=\left[\begin{array}{lll}p & q & r\end{array}\right]^{T}$ denotes the vector of the attitude angular rates, and $I_{b}$ is the inertia matrix of the rigid body, which is expressed below:

$$
I_{b}=\left[\begin{array}{ccc}
I_{x x} & 0 & -I_{x z} \\
0 & I_{y y} & 0 \\
-I_{z x} & 0 & I_{z z}
\end{array}\right] .
$$

The forces and moments, expressed in the BFF, are contributed by gravity, the propulsion system, aerodynamics, and gyroscopic effect caused by rotor tilting action. They can be expressed as

$$
\begin{aligned}
F^{b} & =F_{g}^{b}+F_{p}^{b}+F_{a}^{b}, \\
M^{b} & =M_{p}^{b}+M_{a}^{b}+M_{\mathrm{gy}}^{b},
\end{aligned}
$$

where all the component forces and moments take the form presented below.

2.1. Gravity. The gravity in the BFF can be expressed as

$$
F_{g}^{b}=R_{g}^{b} G^{T}=\left[\begin{array}{lll}
-m g s \theta & m g c \theta s \phi & m g c \theta c \phi
\end{array}\right]^{T} .
$$

\subsection{Propulsion System}

2.2.1. Thrust Vector. The thrust vector can be decomposed along the BFF by using the rotation matrices between the $\mathrm{AFs}$ and BFF: 


$$
\begin{aligned}
F_{p}^{b} & =\sum_{i=1}^{4} R_{t i}^{b} T_{i}^{a}, \\
T_{i}^{a} & =\left[\begin{array}{lll}
0 & 0 & -T_{i}
\end{array}\right]^{T}, \\
T_{i} & =g_{T i}\left(\delta_{i}\right),
\end{aligned}
$$

where $T_{i}^{a}$ are the thrust vectors expressed in the AFs, $T_{i}$ are the thrust values, $\delta_{i}$ are the motor throttles, and $g_{T i}(\cdot)$ represent the mathematical relationships determined by experiments.

2.2.2. Moment Vector. The moment vector generated by the propulsion systems can be expressed as

$$
\begin{aligned}
M_{p}^{b} & =M_{p t}^{b}+M_{p d}^{b}, \\
M_{p t}^{b} & =\sum_{i=1}^{4}\left(d_{i} \times\left(R_{t i}^{b} T_{i}^{a}\right)\right), \\
M_{p d}^{b} & =\sum_{i=1}^{4} R_{t i}^{b} \tau_{i}^{a}, \\
\tau_{i}^{a} & =\left[\begin{array}{lll}
0 & 0 & (-1)^{i} \tau_{i}
\end{array}\right]^{T}, \\
\tau_{i} & =g_{\tau i}\left(\delta_{i}\right),
\end{aligned}
$$

where $M_{p t}^{b}$ is the moment vector generated by the thrust vector, $d_{i}$ are position vectors from the CoG to the axis of each rotor, $\tau_{i}$ represent the torques caused by rotor air resistance, $\tau_{i}^{a}$ are the torque vectors expressed in the AFs, and $M_{p d}^{b}$ is the torque vector expressed in the BFF.

2.3. Gyroscopic Torque. The spinning rotor will cause the gyroscopic precession torque effect. The expression for this moment vector is given as follows:

$$
M_{g y}^{b}=\sum_{i=1}^{4}\left(\omega^{b} \times J_{r i} R_{t i}^{b} \omega_{i}\right),
$$

where $J_{r i}$ are the moments of inertia of the propulsion groups about their axes and $\omega_{i}$ represent the rotation angular velocity vectors of the rotors in the AFs.

2.4. Aerodynamic Force and Moment. Different from reference [27], the rudder is considered in this paper, and the aerodynamic force and moment of the TRUAV are presented as follows:

$$
\begin{aligned}
& F_{a}^{b}=\left[\begin{array}{lll}
-D & Y & -L
\end{array}\right]^{T}=Q S\left[\begin{array}{ll}
-C_{D} & \left.C_{Y}-C_{L}\right]^{T}, \\
& =Q S\left[\begin{array}{c}
C_{X}(\alpha)+C_{X q}(\alpha) \frac{\bar{c}}{2 V} q+C_{X \delta_{e}}(\alpha) \delta_{e}+C_{X \delta_{r}}(\alpha) \delta_{r} \\
C_{Y 0}+C_{Y \beta} \beta+C_{Y p} \frac{\bar{b}}{2 V} p+C_{Y r} \frac{\bar{b}}{2 V} r+C_{Y \delta_{a}} \delta_{a}+C_{Y \delta_{r}} \delta_{r}
\end{array}\right], \\
M_{a}^{b}= & {\left[\begin{array}{c}
l \\
C_{Z}(\alpha)+C_{Z q}(\alpha) \frac{\bar{c}}{2 V} q+C_{Z \delta_{e}}(\alpha) \delta_{e}
\end{array}\right]^{T}=Q\left[C_{l} C_{m} C_{n}\right]^{T},} \\
b\left(C_{l 0}+C_{l \beta} \beta+C_{l p} \frac{\bar{b}}{2 V} p+C_{l r} \frac{\bar{b}}{2 V} r+C_{l \delta_{a}} \delta_{a}+C_{l \delta_{r}} \delta_{r}\right) \\
\bar{c}\left(C_{m 0}+C_{m \alpha} \alpha+C_{m q} q \frac{\bar{b}}{2 V} \bar{c}+C_{m \delta_{e}} \delta_{e}\right) \\
=
\end{array}\right], \\
& \bar{b}\left(\begin{array}{c}
\left.C_{n 0}+C_{n \beta} \beta+C_{n p} \frac{\bar{b}}{2 V} p+C_{n r} \frac{\bar{b}}{2 V} r+C_{n \delta_{a}} \delta_{a}+C_{n \delta_{r}} \delta_{r}\right)
\end{array}\right]
\end{aligned}
$$

where $Q$ is the dynamic pressure, $\alpha$ is the angle of attack, $\beta$ is the sideslip angle, and $\delta_{a}, \delta_{e}$, and $\delta_{r}$ are the deflection angles of the aileron, elevator, and rudder, respectively. Aerodynamic derivations can be obtained by CFD simulations and wind tunnel tests.
In addition, if $\zeta=\left[\begin{array}{lll}x & y & z\end{array}\right]^{T}$ represents the position vector of the aircraft in the EFF. According to the transformation relationship between coordinate systems, the translation kinematic equations and the rotation kinematic equations can be expressed as 


$$
\begin{aligned}
& \dot{\zeta}=R_{b}^{g} V^{b}=\left(R_{g}^{b}\right)^{T} V^{b}, \\
& \dot{\phi}=p+(r c \phi+q s \phi) \tan \theta, \\
& \dot{\theta}=q c \phi-r s \phi \\
& \dot{\psi}=\sec \theta(r c \phi+q s \phi) .
\end{aligned}
$$

In conclusion, based on the aforementioned analysis, the 6-DoF nonlinear dynamics equation of the system can be described in the form of a first-order vector differential equation:

$$
\dot{x}=f(x(t), u(t), t),
$$

where $x$ is the vector of state variables $u, v, w, p, q, r, \phi, \theta, \psi, x_{g}, y_{g}, z_{g}$, and $u$ is the vector of input variables that usually consist of motor throttles $\delta_{1}, \delta_{2}, \delta_{3}$, and $\delta_{4}$ and control surface deflections $\delta_{e}, \delta_{a}$, and $\delta_{r}$, which are considered in transition mode and airplane mode.

2.5. Identification of Parameters. Through the analysis and derivation of the abovementioned formula, the mathematical model is established. However, many parameters need to be identified. In this section, we mainly measure the pull and torque coefficients of the motor-rotor combination by experiments (shown in Figure 3).

A device equipped with a $6-\mathrm{DoF}$ torque/force sensor is used for identifying the mappings between the throttle and the generated thrust and torque. The experimental results are represented in Figures 4 and 5.

Thus, the following polynomial models can be obtained through data fitting:

$$
\begin{aligned}
\left\|T_{1,2}\right\| & =-60.08 \delta^{3}+118.1 \delta^{2}+37.26 \delta \\
\left\|T_{3,4}\right\| & =-44.21 \delta^{3}+91.14 \delta^{2}-1.08 \delta \\
\left\|\tau_{1,2}\right\| & =-2.133 \delta^{3}+4.194 \delta^{2}+1.323 \delta \\
\left\|\tau_{3,4}\right\| & =-1.003 \delta^{3}+2.069 \delta^{2}-0.025 \delta .
\end{aligned}
$$

The aircraft's nominal mass is $15.5 \mathrm{~kg}$. Through a highdetail UG model, we also obtain inertia moments of the body and propulsion groups. The UAV parameters are shown in Table 1.

\section{Design of Controller}

3.1. Control Allocation Strategy. In this part, a control allocation method based on the channel throttles and trim calculations is proposed for the new TRUAV. First, the relationships between the virtual control inputs of the four channels and the actual throttles of the thrusters are established as follows:

$$
\left\{\begin{array}{l}
\delta_{1}=\delta_{H 1}+\delta_{\theta 1}+\delta_{\phi 1}+\delta_{\psi 1} \\
\delta_{2}=\delta_{H 2}+\delta_{\theta 2}+\delta_{\phi 2}+\delta_{\psi 2} \\
\delta_{3}=\delta_{H 3}+\delta_{\theta 3}+\delta_{\phi 3}+\delta_{\psi 3} \\
\delta_{4}=\delta_{H 4}+\delta_{\theta 4}+\delta_{\phi 4}+\delta_{\psi 4}
\end{array}\right.
$$

where $\delta_{H i}, \delta_{\theta i}, \delta_{\phi i}, \delta_{\psi i}(i=1,2,3,4)$ are the weights of the throttles of the four channels described in Table 2 .

When the aircraft is in an equilibrium state, namely, $V^{b}=\dot{V}^{b}=\omega^{b}=\dot{\omega}^{b}=0$, we can easily get the following perturbation equilibrium equations based on the nonlinear dynamics equations (11):

$$
\left\{\begin{array}{l}
\Delta\left(F_{z}^{b}\right)=0, \\
\Delta\left(M^{b}\right)=0,
\end{array}\right.
$$

where $\Delta(\cdot) \triangleq(\cdot)-(\cdot)_{o p}$, "op" denotes the operation point.

According to the description above, the trimmed conditions of the four channels can be expressed as

$$
\left\{\begin{array}{l}
\text { Vertical }: \Delta\left(M_{x}^{b}\right)=\Delta\left(M_{y}^{b}\right)=\Delta\left(M_{z}^{b}\right)=0, \Delta\left(F_{z}^{b}\right) \neq 0, \\
\text { Pitch }: \Delta\left(M_{x}^{b}\right)=\Delta\left(M_{y}^{b}\right)=\Delta\left(F_{z}^{b}\right)=0, \Delta\left(M_{y}^{b}\right) \neq 0, \\
\text { Roll : } \Delta\left(M_{y}^{b}\right)=\Delta\left(M_{z}^{b}\right)=\Delta\left(F_{z}^{b}\right)=0, \Delta\left(M_{x}^{b}\right) \neq 0, \\
\text { Yaw }: \Delta\left(M_{x}^{b}\right)=\Delta\left(M_{y}^{b}\right)=\Delta\left(F_{z}^{b}\right)=0, \Delta\left(M_{z}^{b}\right) \neq 0 .
\end{array}\right.
$$

Before solving the equations, the following assumptions need to be made. (1) In helicopter mode, the efficiency of the aerodynamic effectors and the gyroscopic effect are very low, so they can be ignored. (2) Let $\varepsilon, \theta$, and $\phi$ be equal to zero in helicopter mode. (3) Ignore the smaller higher-order terms, namely,

$$
\begin{aligned}
\Delta\left(T_{i}\right) & =k_{T i} \delta_{i}, \\
\Delta\left(\tau_{i}\right) & =k_{\tau i} \delta_{i},
\end{aligned}
$$

where $k_{T i}=\partial g_{T i} /\left.\partial \delta_{i}\right|_{\delta=\delta_{o p}}$ and $k_{\tau i}=\partial g_{\tau i} /\left.\partial \delta_{i}\right|_{\delta=\delta_{o p}}$.

The 1st and 2nd motors reach the maximum capacity first due to the asymmetric geometric parameters; thus, the weight coefficient of each channel can be obtained based on the 1st motor. The calculation results are presented as follows:

$$
\left\{\begin{array}{l}
\delta_{H 1}=\delta_{H 2}=\delta_{H} \\
\delta_{H 3}=\delta_{H 4}=-\frac{k_{T 1} \cdot d_{1 x}-k_{\tau 1} \cdot \tan \eta}{k_{T 3} \cdot d_{3 x}+k_{\tau 3} \cdot \tan \eta} \delta_{H} \\
\delta_{\theta 1}=\delta_{\theta 2}=-\delta_{\theta} \\
\delta_{\theta 3}=\delta_{\theta 4}=\frac{k_{T 1}}{k_{T 3}} \delta_{\theta} \\
\delta_{\phi 1}=-\delta_{\phi 2}=-\delta_{\phi}, \\
\delta_{\phi 3}=-\delta_{\phi 4}=-\frac{k_{T 1} \cdot \tan \eta \cdot d_{1 x}+k_{\tau 1}}{k_{T 3} \cdot \tan \eta \cdot d_{3 x}+k_{\tau 3}} \delta_{\phi} \\
\delta_{\psi 1}=-\delta_{\psi 2}=-\delta_{\psi}, \\
\delta_{\psi 3}=-\delta_{\psi 4}=\frac{k_{T 1}\left(d_{1 y}+d_{1 z} \tan \eta\right)}{k_{T 3}\left(d_{3 y}+d_{3 z} \tan \eta\right)} \delta_{\psi}
\end{array}\right.
$$




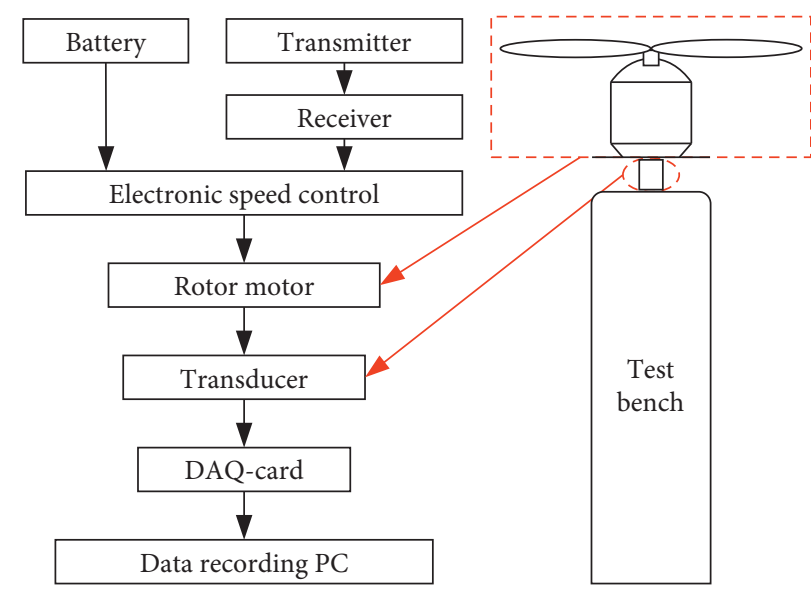

FIGURE 3: Left: scheme of the measurement chain. Right: schematic diagram of the testbed installation. DAQ: data acquisition.
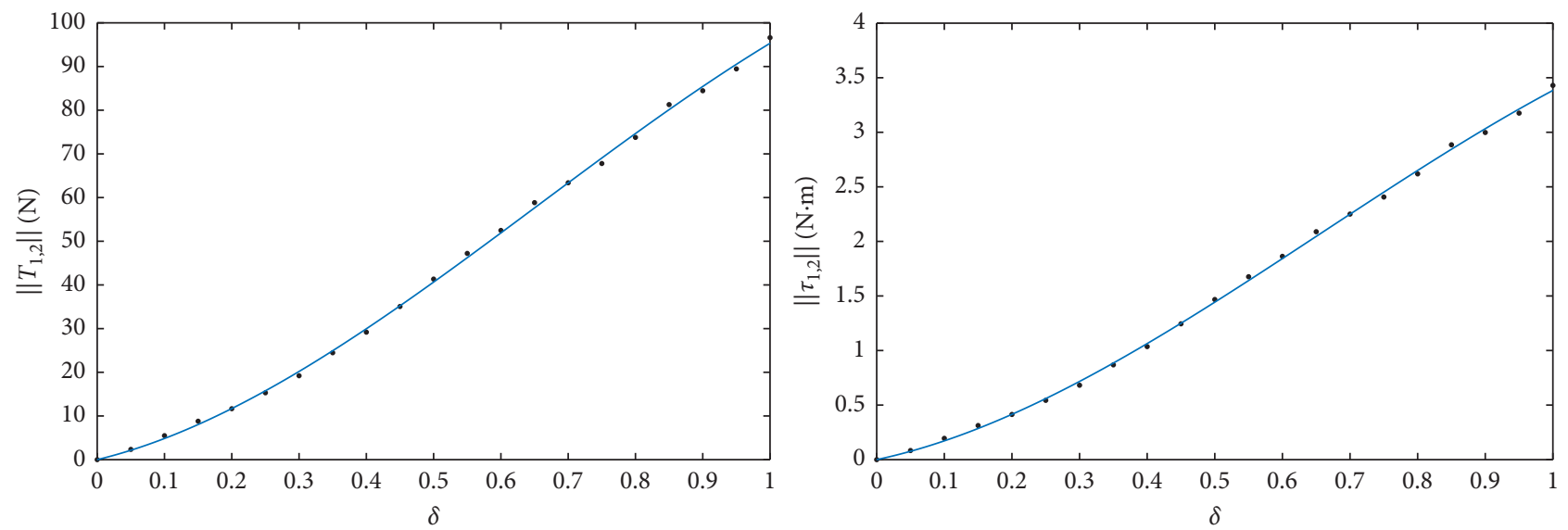

FIgURE 4: Measured values of the thrust and torque of the front motors versus the throttle $\delta$.
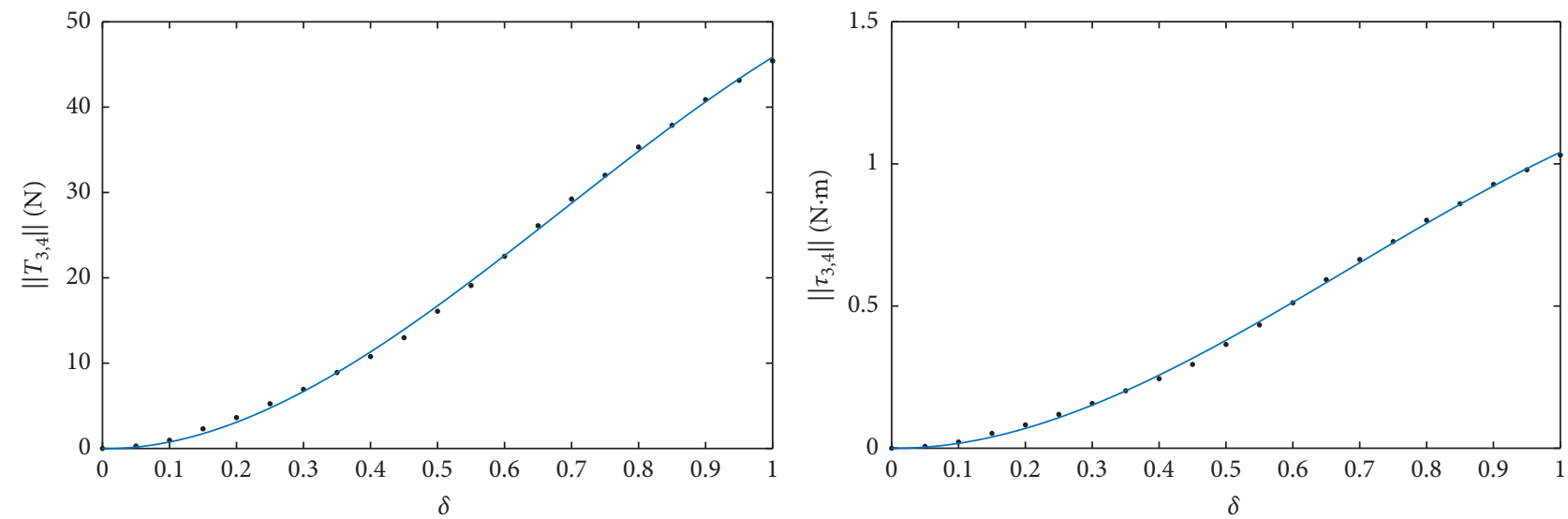

Figure 5: Measured values of the thrust and torque of the back motors versus the throttle $\delta$.

Finally, the control allocation relationships between the channel inputs and the actual throttles can be obtained by substituting equation (17) into equation (13).
3.2. Linearized Model. To design the attitude and altitude controllers in helicopter mode, the nonlinear model (11) is partitioned, taking into account the state vector 
TABLE 2: Description of the channel throttles.

\begin{tabular}{|c|c|}
\hline Channels & Definitions \\
\hline Vertical $\delta_{H} \in[0,1]$ & $\delta_{H}=0$ : motors stop working. $\delta_{H}=1$ : the vertical maximum thrust is generated \\
\hline Pitch $\delta_{\theta} \in[-1,1]$ & $\begin{array}{c}\delta_{\theta}=-1(\text { or }+1) \text { is the maximum maneuvering capability of making aircraft raise up (or down) its nose. } \delta_{\theta}=0 \text { means } \\
\text { no pitching control }\end{array}$ \\
\hline $\begin{array}{l}\text { Roll } \delta_{\phi} \in[-1,1] \\
\text { Yaw } \delta_{\psi} \in[-1,1]\end{array}$ & $\begin{array}{l}\left.\delta_{\phi}=-1 \text { (or }+1\right) \text { is the maximum maneuvering capability of rolling left (or right). } \delta_{\theta}=0 \text { means no rolling control } \\
\delta_{\psi}=-1(\text { or }+1) \text { is the maximum maneuvering capability of yawing left (or right). } \delta_{\psi}=0 \text { means no yawing control }\end{array}$ \\
\hline
\end{tabular}

$\widehat{x}=\left[\begin{array}{llllllll}p & q & r & w & \phi & \theta & \psi & z_{g}\end{array}\right]^{T}$ and the input vector $\widehat{u}=\left[\begin{array}{llll}\delta_{H} & \delta_{\phi} & \delta_{\theta} & \delta_{\psi}\end{array}\right]^{T}$, which leads to a new system:

$$
\dot{\hat{x}}=f(\widehat{x}(t), \widehat{u}(t), t) \text {. }
$$

Since the controllers are designed based on linear models, system (18) is linearized around the equilibrium operation point, expanding it through Taylor series and truncating in the first-order terms. Thus, the linearized model is presented below:

$$
\dot{\hat{x}}=A \widehat{x}+B \widehat{u},
$$

where $A=\partial f /\left.\partial \widehat{x}\right|_{\widehat{u}=\widehat{u}_{o p}} ^{\widehat{x}=\widehat{x}_{o p}}$ and $B=\partial f /\left.\partial \widehat{u}\right|_{\widehat{u}=\widehat{u}_{o p}} ^{\widehat{x}=\widehat{x}_{o p}}$.

3.3. RSLQR Control. Based on model (19), the following linearized system equation for the TRUAV is given by

$$
\left\{\begin{array}{l}
\dot{\hat{x}}=A \widehat{x}+B \widehat{u} \\
y_{c}=C_{c} \widehat{x}
\end{array}\right.
$$

where $y_{c}$ is the output vector and $C_{c}$ is the corresponding transition matrix.

The RSLQR control method is developed based on the LQR method by introducing state deviation into the system as a new variable. In this way, the deviation can be adjusted to zero so that the system state variable can accurately track the input command. If the deviation is supposed to be $e=r_{c}-y_{c}$, where $r_{c}$ is the control instruction. The new state equation can be defined as

$$
\dot{z}=\widetilde{A} z+\widetilde{B} \mu
$$

where $z=\left[\begin{array}{ll}e & \dot{\hat{x}}\end{array}\right]^{T}, \mu=\dot{\hat{u}}, \widetilde{A}=\left[\begin{array}{cc}0 & -C_{c} \\ 0 & A\end{array}\right]$, and $\widetilde{B}=\left[\begin{array}{l}0 \\ B\end{array}\right]$.

The cost function of the RSLQR control based on the new system is given by

$$
J=\frac{1}{2} \int_{0}^{\infty}\left(z(t)^{T} \mathrm{Q} z(t)+\mu(t)^{T} R \mu(t)\right) \mathrm{d} t,
$$

where $Q$ and $R$ are the weight matrices. The weighting matrices are chosen to characterize the performance of the control design. The techniques available for choosing these matrices are still immature. To facilitate engineering applications, matrix $R$ is chosen as the identity matrix and matrix $Q$ is selected as the diagonal matrix according to the requirements of the control system. The values in matrix $Q$ are scaled using Bryson's law in reference [28]. Finally, the gain matrix of the RSLQR control can be expressed as

$$
K_{c}=\left[\begin{array}{ll}
k_{i} & k_{p}
\end{array}\right]=R^{-1} B^{T} P
$$

where $k_{i}$ and $k_{p}$ are the different blocks, which have the same dimensions as $e$ and $\hat{x}$, and $P\left(=P^{T}>0\right)$ is the solution of the following algebraic Riccati equation:

$$
P A+A^{T} P-P B R^{-1} B^{T} P+Q=0 .
$$

The optimal control input of the RSLQR control is shown in equation (25), and a schematic diagram of the RSLQR state feedback control is presented in Figure 6:

$$
\begin{aligned}
\widehat{u} & =\int \mu d t=-K_{c} \int z d t=-k_{i} \int e \mathrm{~d} t-k_{p} \int \dot{\hat{x}} \mathrm{~d} t \\
& =-k_{i} \int e \mathrm{~d} t-k_{p} \widehat{x} .
\end{aligned}
$$

As seen from the diagram, the RSLQR control improves the system type and enhances the antijamming ability because it adds the integral element in the state feedback control loop. Therefore, the flight control law, based on the RSLQR control, can improve the dynamic behavior and the steady-state performance of the control system. Considering system (20) again, we can extract angular rate models and a vertical velocity model. Then, the RSLQR controllers are designed for the angular rate and velocity loops, which are also called inner loops. Furthermore, to meet the higher control precision demand, the mature and reliable PI control method is used for attitude and height loop control. Taking the roll channel as an example, the control structure is shown in Figure 7, where PI gains $k_{p}^{\phi}$ and $k_{i}^{\phi}$ are determined by the optimum damping ratio and bandwidth matching relationship between the inner and outer loop. They can be obtained through the PI parameter tuning toolbox in MATLAB.

3.4. RSLQR Control Based on ESO. The designed controllers based on the RSLQR control do not work well under uncertainty and disturbance conditions because it is not possible to obtain the real states all the time. In this section, an ESO is designed to estimate the states and the unknown total disturbances. Then, the final control input is obtained by compensating for the initial control quantity of RSLQR, which can effectively improve the ability to withstand disturbances and uncertain factors.

Considering the addition of a disturbance term to system (18), the following second-order nonlinear system can be obtained:

$$
\left\{\begin{array}{l}
\dot{x}_{1}=x_{2}, \\
\dot{x}_{2}=b \bar{u}+f(\cdot), \\
y=x_{1},
\end{array}\right.
$$




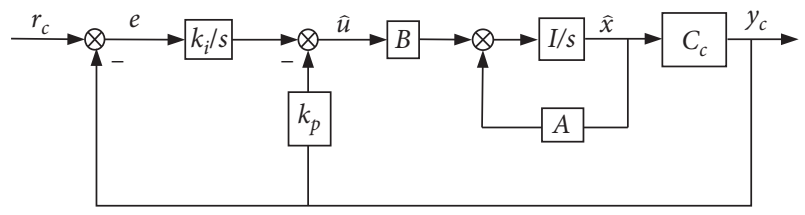

FIgURE 6: Schematic diagram of the RSLQR control.

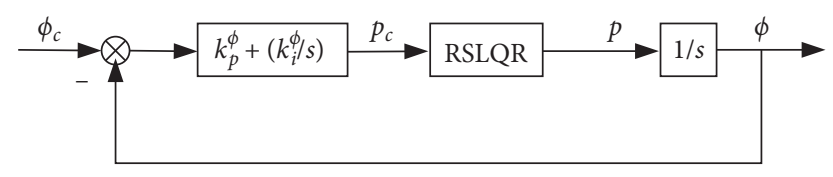

FIgURE 7: Structure of roll angle control.

where $x_{1}$ represents the state variable of attitude or height, $f(\cdot)$ is the total disturbance of the system including the model uncertainty and the external interference, $\bar{u}$ denotes the control input, and $b$ is the magnification. Then, the total disturbance is expanded into a new state variable $x_{3}$, and let $\dot{x}_{3}=\varsigma$. The new system can be expressed as follows:

$$
\left\{\begin{array}{l}
\dot{x}_{1}=x_{2}, \\
\dot{x}_{2}=x_{3}+b u, \\
\dot{x}_{3}=\varsigma \\
y=x_{1} .
\end{array}\right.
$$
[29]:

For the extended system, the following ESO is designed

$$
\left\{\begin{array}{l}
e=z_{1}-y, \\
\dot{z}_{1}=z_{2}-\beta_{1} e, \\
\dot{z}_{2}=z_{3}-\beta_{2} f a l\left(e, \alpha_{1}, \delta\right)+b \bar{u}, \\
\dot{z}_{3}=-\beta_{3} f a l\left(e, \alpha_{2}, \delta\right),
\end{array}\right.
$$

where $Z=\left[\begin{array}{lll}z_{1} & z_{2} & z_{3}\end{array}\right]^{T}$ is the estimation of the extended state vector $X=\left[\begin{array}{lll}x_{1} & x_{2} & x_{3}\end{array}\right]^{T}, \beta_{1}, \beta_{2}$, and $\beta_{3}$ are the observer gains, and the nonlinear function $f a l\left(e, \alpha_{i}, \delta\right)$ is expressed as

$$
f a l\left(e, \alpha_{i}, \delta\right)= \begin{cases}\frac{e}{\delta^{1-\alpha_{i}},}, & |e| \leq \delta, \\ |e|^{\alpha_{i}} \operatorname{sgn}(e), & |e|>\delta,\end{cases}
$$

where $\alpha_{1}, \alpha_{2}$, and $\delta$ are parameters of the nonlinear function fal (.). $\alpha_{i}$ determines the nonlinear shape, and generally $\alpha_{1}=$ 0.5 and $\alpha_{2}=0.25$. $\delta$ determines the width of its linear interval around the origin. If it is too large, the disturbance signal with large amplitude cannot be tracked. In contrast, high-frequency oscillations near the origin easily appear. The observer parameters $\beta_{i}$ determine the tracking speed of states $x_{i}$. Furthermore, these parameters are mutually constrained. Appropriate increase of $\beta_{1}$ and $\beta_{2}$ can effectively suppress the observer oscillation caused by excessive $\beta_{3}$. Therefore, the tuning of these three parameters should be coordinated. By continuously adjusting these parameters, the observer can observe the states well in real time, especially the expanded state $x_{3}$. The detailed tuning method can be found in reference [30]. The control structure is shown in Figure 8.

After obtaining the estimated value $z_{3}$ of total disturbance, the real-time control quantity can be obtained through disturbance compensation for the RSLQR controller output $\widehat{u}$, namely,

$$
\bar{u}=\widehat{u}-\frac{z_{3}}{b} .
$$

By substituting equation (30) into system (25), the nonlinear control system becomes a linear integral series system, which is called dynamic compensation linearization [31]:

$$
\left\{\begin{array}{l}
\dot{x}_{1}=x_{2} \\
\dot{x}_{2}=b \widehat{u} \\
y=x_{1}
\end{array}\right.
$$

\section{Simulation Results}

In this section, some results are presented to illustrate the controller efficiency considering the environment parameter $g=9.8\left(\mathrm{~m} / \mathrm{s}^{2}\right)$. The TRUAV parameters are shown in Table I. The steady-state vector of hovering flight is selected as $\widehat{x}_{0}=\left[\begin{array}{llllllll}0 & 0 & 0 & -0.1 & 0 & 0 & 0 & -20\end{array}\right]$. By substituting the parameters into system (17) and balancing the equations, we get the following equilibrium states $\hat{x}_{o p}=\left[\begin{array}{llllllll}0 & 0 & 0 & -0.097 & 0 & 0 & 0 & -20\end{array}\right]$ and equilibrium inputs

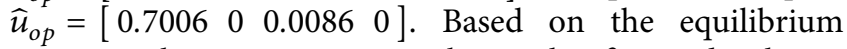
operational points, we can obtain the first-order linear models of the four channels, namely, $A_{w}=$ $-0.2993, B_{w}=-20.46 ; \quad A_{P}=-0.2993, \quad B_{P}=-65.1$; $A_{q}=-0.2993, B_{q}=-86.92$; and $A_{r}=-0.2993, B_{r}=-20.16$. The corresponding weight matrices are chosen as $Q_{w}=$ $\operatorname{diag}\left(\left[\begin{array}{ll}5 & 0.5\end{array}\right]\right), \quad Q_{p}=\operatorname{diag}\left(\left[\begin{array}{ll}70 & 0.4\end{array}\right]\right), \quad Q_{q}=\operatorname{diag}$ ([ $\left.\left.\begin{array}{ll}80 & 0.6\end{array}\right]\right), Q_{r}=\operatorname{diag}\left(\left[\begin{array}{ll}5 & 0.5\end{array}\right]\right)$, and $R_{\mathrm{i}}=1(i=w, p, q, r)$. Then, the RSLQR control gains and PI gains are obtained by using the MATLAB toolbox, and the results are shown in Table 3. Furthermore, according to the parameter tuning rules mentioned above, the ESO parameters are set as $\alpha_{1}=0.5, \quad \alpha_{2}=0.25, \delta=0.5, \quad \beta_{1}=200, \quad \beta_{2}=1864, \quad$ and $\beta_{3}=14220$.

In the first described simulations, disturbance is not considered. For simplicity, this paper takes the vertical channel as an example to show the controller performance. In addition, the RSLQR controller is compared with the PID controller to demonstrate its advantages in the real flight test. The hovering height is set to $3.5 \mathrm{~m}$. The simulation results are shown in Figures 9 and 10. The time and frequency domain attributes of this controller are given in Table 4. Figure 9 shows that the PID and RSLQR controllers can meet the system requirements, but the RSLQR controller does not exhibit overshoot and is gentler than the PID controller in the same response time. Figure 10 shows that the RSLQR controller produces a smaller control input in the initial response, which avoids the phenomenon of larger angle rates caused by servo command mutation and protects 


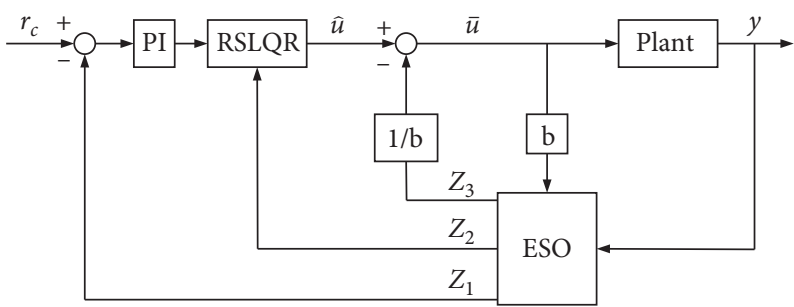

FIgURE 8: Structure of the RSLQR control based on ESO.

TABLE 3: The control gains of the four channels.

\begin{tabular}{cccccc}
\hline & Gains & Vertical channel & Pitch channel & Roll channel & Yaw channel \\
\hline \multirow{2}{*}{ RSLQR } & $k_{i}$ & 2.2403 & 8.9443 & 8.3666 & 2.2361 \\
& $k_{p}$ & -0.8412 & -0.7749 & -0.8060 & -0.8349 \\
\hline \multirow{2}{*}{ PI } & $k_{i}$ & 0.013 & 0.353 & 0.304 & 0.011 \\
& $k_{p}$ & 0.865 & 4.612 & 4.288 & 0.809 \\
\hline
\end{tabular}

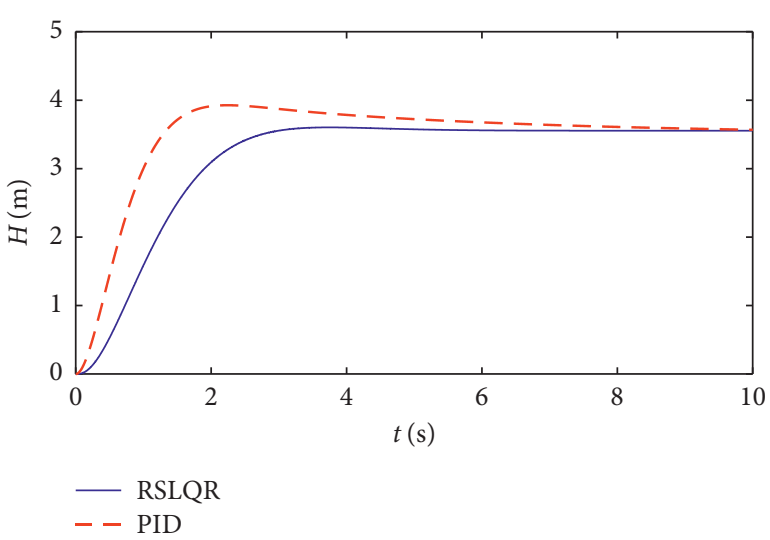

Figure 9: Comparison results of height response.

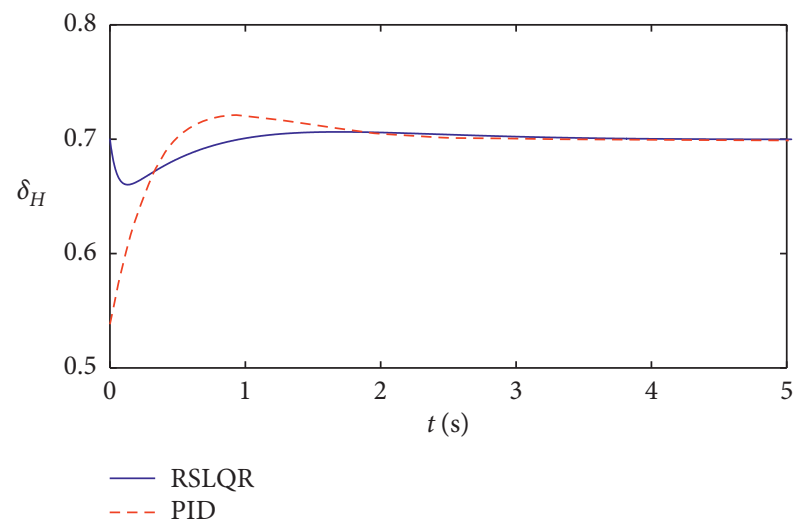

Figure 10: Comparison results of control input.

the actuators from saturation. Thus, the RSLQR control shows a higher overload resistance capability and a better performance than the PID control.

In the following described simulations, disturbance is considered. In the actual flight, the disturbance mainly comes from air convection [31], which is a random low- frequency disturbance. In addition, the high-frequency noise interference in the output measurement link is not considered in this paper. The reason is that the large observer gains $\beta_{i}$ in the ESO will amplify the measurement noise, which will cause a bad effect on the performance of the observer [32]. To eliminate the influence of noise, we first processed it with a low-pass filter in the actual test. Since the study of filters is not the focus of this paper, for the sake of brevity, it is not discussed here. Therefore, in the simulation test, the disturbance is assumed to be a sinusoidal perturbation and is expressed as $0.5 \sin (10 t)$. Taking the roll channel as an example, the observation effect of ESO is shown in Figure 11.

Figure 11 shows that ESO can accurately estimate the given sinusoidal disturbance, where the observed disturbance amplitude is basically the same as the actual value, and the phase deviation between the observation value and the actual value is within the acceptable range.

Figure 12 shows the response of the roll angle controller with and without ESO under disturbance and disturbancefree conditions. To ensure the stability of the TRUAV, the roll angle should be less than $5^{\circ}$.

It can be seen from the figure that the controller with ESO maintains the quality of its performance compared with the real roll angle controller under disturbance conditions, and moreover, it exhibits a better performance than the controller without ESO under disturbance conditions.

\section{Flight Test Results}

In this part, the TRUAV platform is assembled to perform a preliminary experiment. The partial experimental settings are shown in Figure 13.

The platform consists of the onboard section, which includes the TRUAV frame and an autopilot, and the ground section, which includes a ground station and a remote controller. The autopilot can measure the flight states, send them to the ground station, and then control the flight of the aircraft. The autopilot adopts the Pixhawk autopilot system, which is an independent, open-source, and open-hardware 
TABLE 4: The time and frequency domain attributes of the controller.

\begin{tabular}{lccc}
\hline Time domain characteristics & Rise time & Steady-state error & 0 \\
& $1.66 \mathrm{~s}$ & 0 & Overshoot \\
\hline \multirow{2}{*}{ Frequency domain characteristics } & Phase margin & Cut-off frequency & Magnitude margin \\
& $68.4^{\circ}$ & $0.822 \mathrm{rad} / \mathrm{s}$ & $27.5 \mathrm{db}$ \\
\hline
\end{tabular}

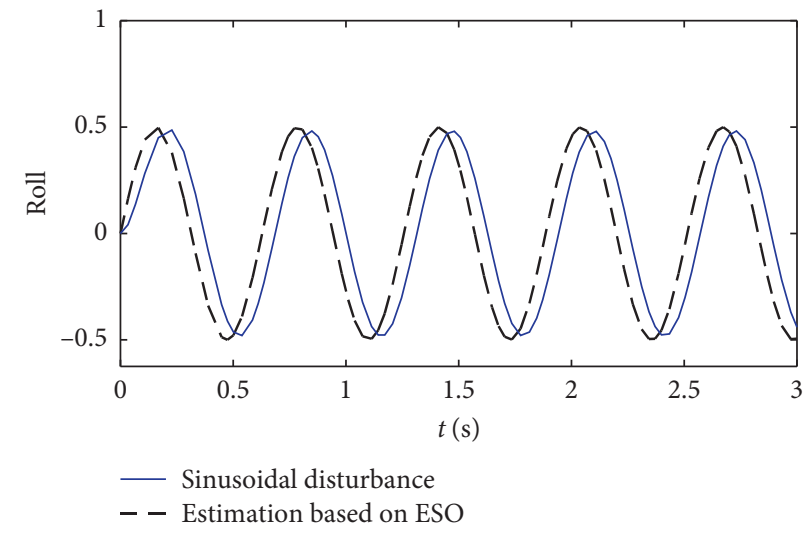

FIGURE 11: Disturbance estimation based on ESO.

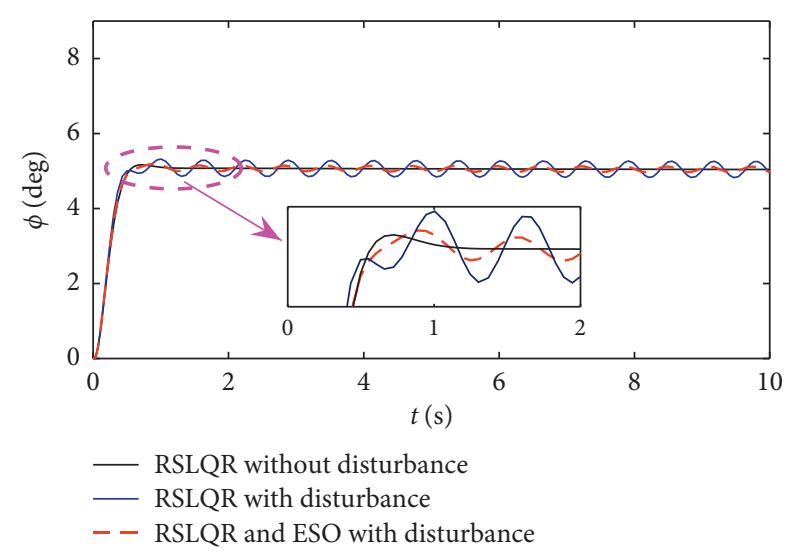

FIGURE 12: Comparison of the roll angle response based on RSLQR under disturbance-free conditions, under disturbance conditions without ESO, and under disturbance conditions with ESO.

project developed by 3DRobotics. Since the Pixhawk opensource hardware platform is relatively mature, we have carried out the secondary development on this basis. The main contents include updated flight control algorithms, added new flight management contents, added new preflight inspection contents, added new emergency management methods, and added new data recording functions. The Pixhawk, running the NuttX real-time operating system, utilizes a $168 \mathrm{MHz}$ CortexM4FARM processor and has $256 \mathrm{~KB}$ of RAM and $2 \mathrm{MB}$ of flash memory. The system has 14 pulse-width modulation servo outputs. The onboard sensors include an IMU, a GPS module with data updated at $5 \mathrm{~Hz}$, a barometer, and a digital airspeed sensor. The aircraft can be controlled by both the autopilot and a pilot. If the pilot finds the TRUAV in an abnormal flight manner, the control is switched to the remote controller mode using a Futaba T14SG $2.4 \mathrm{GHz}$ transmitter to restore the aircraft to safe flight conditions. The ground station adopts the software "QGroundControl," which is open-source ground control software compatible with the Pixhawk autopilot. The ground station is used to observe the flight status of the TRUAV and control it by sending commands to the autopilot through the data link with a radio frequency of $900 \mathrm{MHz}$. To facilitate the parameter tuning and algorithm verification, we have also conducted the related secondary development of the "QGroundControl," mainly including the following: customized some communication protocols, added the parameter settings of the proposed control algorithms, added the options of preflight check, and added the options of emergency disposal. Finally, two sets of hovering tests are carried out in a wide area, as shown in Figure 14.

In the first experiment, the controller is designed based on RSLQR. First, a $3.5 \mathrm{~m}$ altitude step control command is given during the vertical take-off phase, and then the aircraft hovers at this altitude. The test results are illustrated in Figures 15 and 16. From Figure 15 it can be observed that the height can reach the preset value in a short time, and the tracking curve is smooth and gentle. Figure 16 shows that the control input value is approximately 0.7 , with a sufficient margin to avoid the motor saturation problem. Additionally, there is no significant change in the control input as the instruction changes, thereby reducing the available overload requirement of the actuator. The test results are consistent with the simulation results, which verify the effectiveness of the RSLQR controller.

In the following experiment, ESO is considered in the controller design. For the convenience of comparison, taking the roll channel as an example, the test results of the attitude control and the tracking error based on the RSLQR controller with ESO and the RSLQR controller without ESO are presented in Figures 17 and 18, respectively. It can be seen clearly from the figures that the error variation range of the 


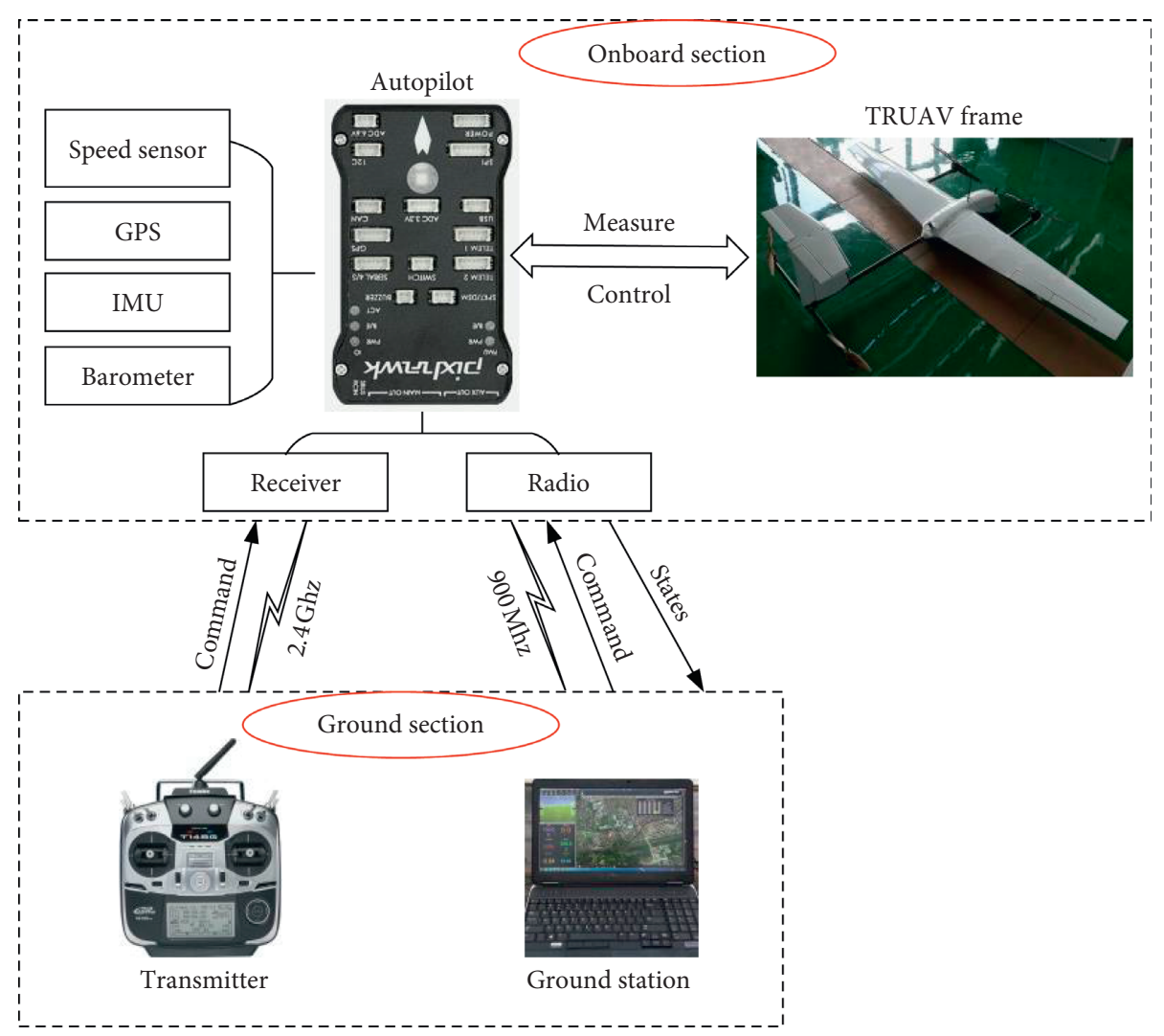

FIGURE 13: Block diagram of the platform.

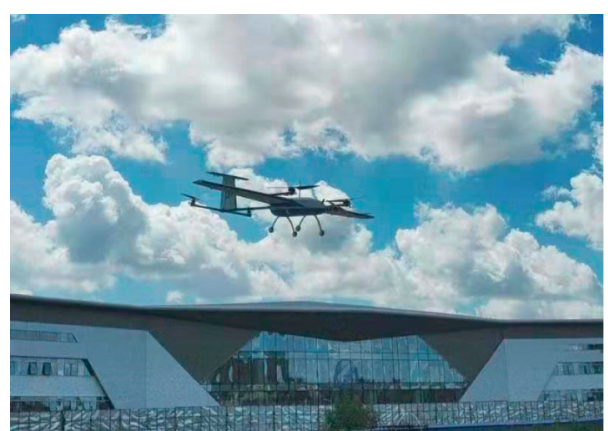

Figure 14: Hovering flight test photo.

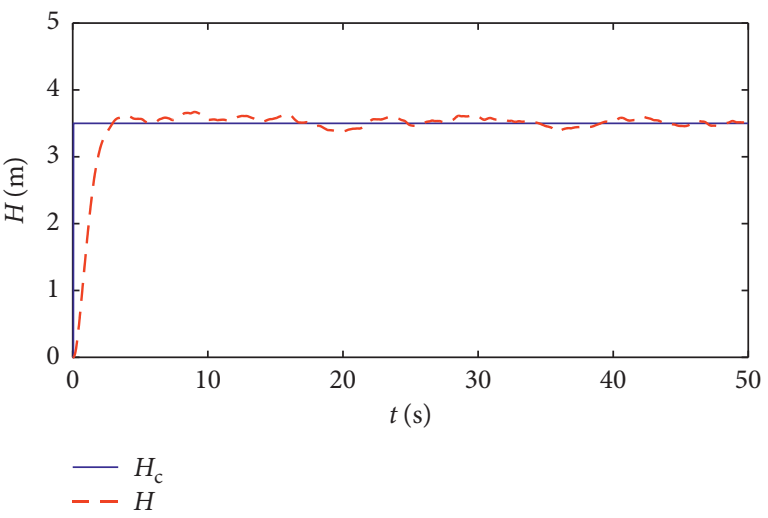

Figure 15: Height control of RSLQR.

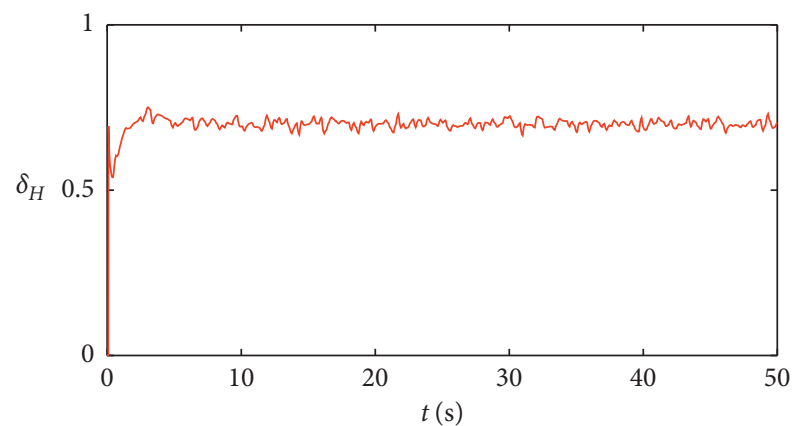

Figure 16: Control input of the vertical channel. 

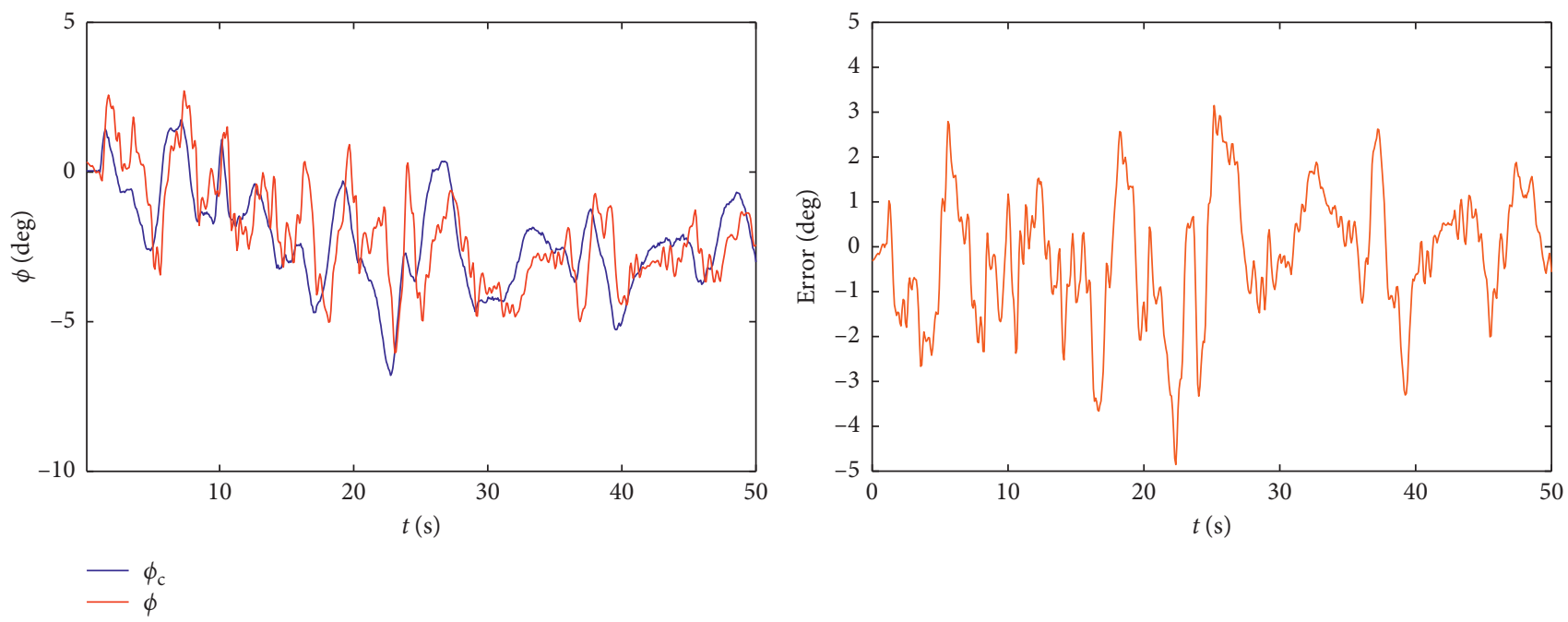

FIgURE 17: Roll control and tracking error of the RSLQR controller with ESO.
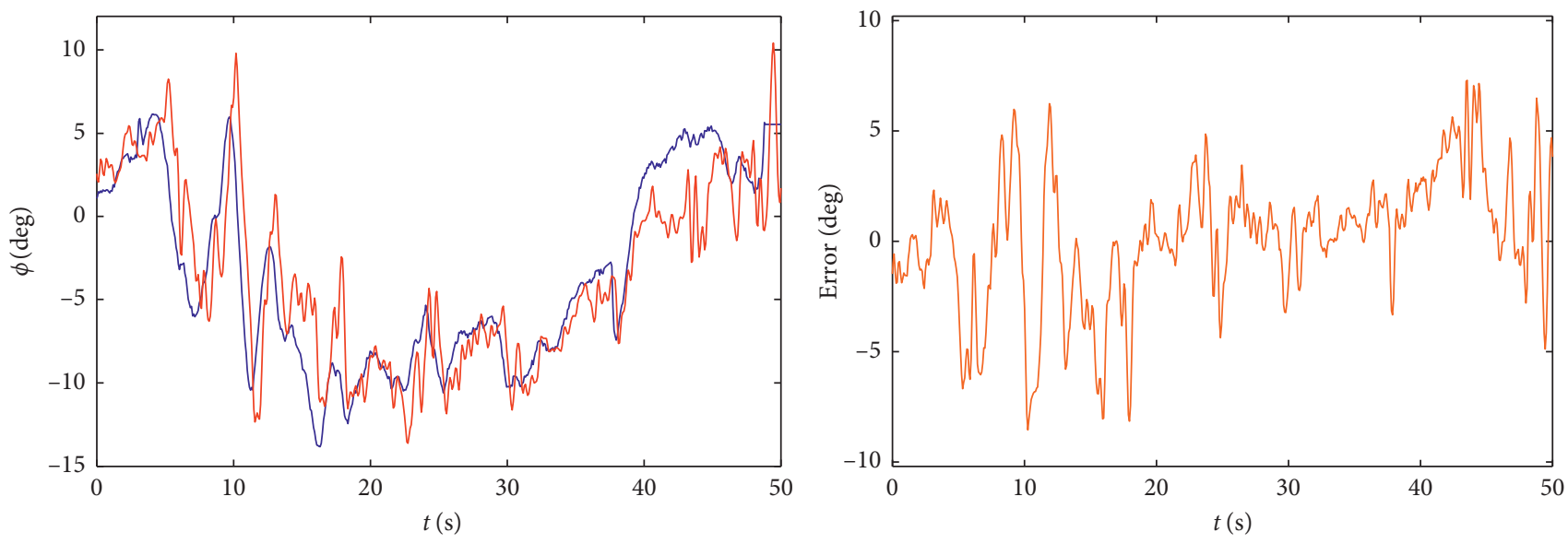

$-\phi_{c}$

FIGURE 18: Roll control and tracking error of the RSLQR controller without ESO.

former roll angle is basically within $\left[-4.8^{\circ}, 3.1^{\circ}\right]$, while the latter is within $\left[-8.5^{\circ}, 7.3^{\circ}\right]$. To help analyze the performance of the controllers, the mean squared error (MSE) performance index is used. The MSE index is relative to the control error with a lower MSE suggesting a better attitude tracking. Through analysis and calculation, the MSE values based on the RSLQR controller with ESO and the RSLQR controller without ESO are 1.9861 and 8.8291, respectively. The attitude angle has a smaller MSE with ESO. Therefore, the RSLQR controller based on ESO can better deal with the disturbance problem during actual flight.

\section{Conclusions and Future Works}

This paper presents the control system design of a new TRUAV. The major contributions of this article are as follows:
(1) A novel configuration scheme of TRUAV is designed. The mathematical model is established, and the related coefficients are identified by designed experiments and numerical analyses.

(2) A control allocation method based on the channel throttles and trim calculations is proposed for the new TRUAV.

(3) An improved control method based on the RSLQR control and ESO is proposed to stabilize the altitude and attitude of the hover phase. Similar to the PID controller, the new linear controller has low cost and is easy to implement in engineering. Meanwhile, it has a higher overload resistance capability, a stronger antijamming ability and a better performance than the PID control.

(4) The simulations and hovering flight tests verify the credibility of this new configuration, the effectiveness 
of the mathematical model and the proposed control strategy, which provide the basis for the next full envelope flight control design.

The proposed control method plays a significant role in the single state point controller design. It provides a research method for the transition control and the fixed-wing flight control. In particular, the transition phase is a multistate point controller design process. Thus, how to realize smooth switching between controllers is a key point. In subsequent research, the following work needs to be performed:

(1) The mixing scheme needs to be explored to perform a smooth gain-scheduling between the proposed controllers

(2) A full envelope flight test is planned to carry out further research studies on the flight control law.

\section{Data Availability}

The data used to support the findings of this study are available from the corresponding author upon request.

\section{Conflicts of Interest}

The authors declare that they have no conflicts of interest.

\section{Acknowledgments}

This work was supported by the Major Program for Science and Technology Development of Jilin: Key Technologies for the Industrialization of Highly Reliable Fixed-wing UAVs (Grant no. 20170201006GX) and the Key Science and Technology Research and Development Projects of Jilin: Research on the Key Technologies of UAV Light and Small Swing Scanning Intelligent Aerial Survey System (Grant no. 20180201106GX).

\section{References}

[1] J. Lee, B. Min, and E. Kim, "Autopilot design of tilt-rotor UAV using particle swarm optimization method," in Proceedings of the 2007 International Conference on Control, Automation and Systems, IEEE, Seoul, Korea, October 2007.

[2] D. Wyatt, Eagle Eye Pocket Guide, Bell Helicopter Textron Inc, Fort Worth, TX, USA, 2004.

[3] S. Choi, Y. Kang, S. Chang, S. Koo, and J. M. Kim, "Development and conversion flight test of a small tilt rotor unmanned aerial vehicle," Journal of Aircraft, vol. 47, no. 2, pp. 730-732, 2010.

[4] F. Kendoul, I. Fantoni, and R. Lozano, "Modeling and control of a small autonomous aircraft having two tilting rotors," in Proceedings of the 44th IEEE Conference on Decision and Control, and the European Control Conference, IEEE, Seville, Spain, December 2005.

[5] Y. G. Song and H. J. Wang, "Design of flight control system for a small unmanned tilt rotor aircraft," Chinese Journal of Aeronautics, vol. 22, no. 3, pp. 250-256, 2009.

[6] A. Alkamachi and E. Erçelebi, " $H_{\infty}$ control of an overactuated tilt rotors quadcopter," Journal of Central South University, vol. 25, no. 3, pp. 586-599, 2018.
[7] F. Senkul and E. Altuğ, "Modeling and control of a novel tiltroll rotor quadrotor UAV," in Proceedings of the International Conference on Unmanned Aircraft Systems (ICUAS), IEEE, Atlanta, GA, USA, May 2013.

[8] E. Cetinsoy, S. Dikyar, C. Hancer et al., "Design and construction of a novel quad tilt-wing UAV," Mechatronics, vol. 22, no. 6, pp. 723-745, 2012.

[9] M. Sato and K. Muraoka, "Flight Controller design and demonstration of quad-tilt-wing unmanned aerial vehicle," Journal of Guidance, Control, and Dynamics, vol. 38, no. 6, pp. 1071-1082, 2015.

[10] C. Papachristos, K. Alexis, and A. Tzes, "Dual-authority thrust-vectoring of a tri-tiltrotor employing model predictive control," Journal of Intelligent \& Robotic Systems, vol. 81, no. 3-4, pp. 471-504, 2016.

[11] A. Vuruskan, B. Yuksek, U. Ozdemir, A. Yukselen, and G. Inalhan, "Dynamic modeling of a fixed-wing VTOL UAV," in Proceedings of the 2014 International Conference on Unmanned Aircraft Systems (ICUAS), May 2014.

[12] G. R. Flores, J. Escareno, R. Lozano, and S. Salazar, "Quadtilting rotor convertible mav: modeling and real-time hover flight control," Journal of Intelligent \& Robotic Systems, vol. 65 , no. $1-4$, pp. 457-471, 2012.

[13] G. Flores, I. Lugo, and R. Lozano, "6-DOF hovering controller design of the quad tiltrotor aircraft," in Proceedings of the 53rd IEEE Annual Conference on Decision and Control, IEEE, Los Angeles, CA, USA, December 2014.

[14] J.-J. Xiong and E.-H. Zheng, "Position and attitude tracking control for a quadrotor UAV," ISA Transactions, vol. 53, no. 3, pp. 725-731, 2014.

[15] Y. C. Yin, H. Niu, and X. Liu, "Adaptive neural network sliding mode control for quad tilt rotor aircraft," Complexity, vol. 2017, Article ID 7104708, 13 pages, 2017.

[16] B.-M. Kim, K. Choi, and B. S. Kim, "Trajectory tracking controller design using neural networks for tiltrotor UAV," in Proceedings of the AIAA-2007-6460 Guidance, Navigation and Control Conference and Exhibit, August 2007.

[17] R. T. Rysdyk and A. J. Calise, "Adaptive model inversion flight control for tilt-rotor aircraft," Journal of Guidance, Control, and Dynamics, vol. 22, no. 3, pp. 402-407, 1999.

[18] B. Yuksek, A. Vuruskan, U. Ozdemir, M. A. Yukselen, and G. Inalhan, "Transition flight modeling of a fixed-wing VTOL UAV," Journal of Intelligent \& Robotic Systems, vol. 84, no. 14, pp. 83-105, 2016.

[19] C. Chen, J. Zhang, D. Zhang, and L. Shen, "Control and flight test of a tilt-rotor unmanned aerial vehicle," International Journal of Advanced Robotic Systems, vol. 14, no. 1, pp. 1-12, 2017.

[20] A. N. Wan, G. M. S. Sim, R. W. L. Koh et al., "Development of UGS-TUM vertical takeoff \& landing (VTOL) drone with flight control," in Proceedings of the 2019 First International Symposium on Instrumentation, Control, Artificial Intelligence, and Robotics (ICA-SYMP), Bangkok, Thailand, January 2019.

[21] Z. Duan, "Robust servo LQR flight control law design of UAVs," Computer Measurement \& Control, vol. 23, no. 8, pp. 2713-2715, 2015.

[22] E. M. Mohamed and L. Yan, "Robust servomechanism LQR comparison with two- and three-Loop autopilot designs," International Journal of Modeling and Optimization, vol. 7, no. 1, pp. 28-33, 2017.

[23] J. Dou, X. Kong, and B. Wen, "Altitude and attitude active disturbance rejection controller design of a quadrotor unmanned aerial vehicle," Proceedings of the Institution of 
Mechanical Engineers, Part G: Journal of Aerospace Engineering, vol. 231, no. 9, pp. 1732-1745, 2017.

[24] Y. Xia, F. Pu, S. Li, and Y. Gao, "Lateral path tracking control of autonomous land vehicle based on ADRC and differential flatness," IEEE Transactions on Industrial Electronics, vol. 63, no. 5, pp. 3091-3099, 2016.

[25] Z. W. Kong and Q. Lu, "Mathematical modeling and modal switching control of a novel tiltrotor UAV," Journal of Robotics, vol. 2018, Article ID 8641731, 12 pages, 2018.

[26] G. V. Raffo, Robust control strategies for a quadrotor helicopter: an underactuated mechanical system, Ph.D. dissertation, Universidad de Sevilla, Seville, Spain, 2011.

[27] C. An, D. Zhang, J. Zhang, and T. Li, "A New structural configuration of tilting rotor unmanned aerial vehicle modeling," in Proceedings of the 2017 Proceedings of the 35th Chinese Control Conference, Chengdu, China, July 2017.

[28] M. A. Johnson and M. J. Grimble, "Recent trends in linear optimal quadratic multivariable control system design," IEE Proceedings D Control Theory and Applications, vol. 134, no. 1, pp. 53-71, 1987.

[29] J. K. Liu, Advanced PID Control and MATLAB Simulation, Publishing House of Electronics Industry, Beijing, China, 2016.

[30] Y. S. Liu, S.-X. Yang, and W. Wang, "An active disturbancerejection flight control method for quad-rotor unmanned aerial vehicles," Control Theory \& Applications, vol. 32, no. 10, pp. 1351-1360, 2015.

[31] J. Pan and C. L. Liu, "UAV attitude control with LQR controller based on extended state observer," Journal of System Simulation, vol. 30, no. 2, pp. 753-759, 2018.

[32] F. Lin, H. Sun, Q. Zheng, and Y. Xia, "Novel extended state observer for uncertain system with measurement noise," Control Theory \& Applications, vol. 6, no. 22, pp. 995-998, 2005. 